\title{
A Coaxial Laser Endoscope with Arbitrary Spots in Endoscopic View for Fetal Surgery
}

\author{
Noriaki Yamanaka $^{1}$, Hiromasa Yamashita ${ }^{1}$, Ken Masamune $^{1}$, Hongen Liao $^{2}$, \\ Toshio Chiba ${ }^{3}$, and Takeyoshi Dohi ${ }^{1}$ \\ 1 Graduate School of Information Science and Technology, The University of Tokyo \\ ${ }^{2}$ Graduate School of Engineering, The University of Tokyo \\ 3 Clinical Research and Development, National Center for Child Health and \\ Development
}

\begin{abstract}
In this paper, we describe a rigid endoscope that transmits a laser beam coaxially to arbitrary points in the endoscopic view, mainly for treatment of twin-to-twin transfusion syndrome. The endoscope consists of a hotmirror for coaxial transmission of visible light and a Nd:YAG laser beam, and galvanometers for controlling the beam irradiation angle. We evaluated the transmission efficiency of the laser power, the spot size through the endoscope and accuracy in positioning the beam. The maximum laser transmission efficiency was $39 \%$ and the spot diameter was $2.2-3.2 \mathrm{~mm}$ at a distance of $10-20 \mathrm{~mm}$. The positioning accuracy was mostly within $1.0 \mathrm{~mm}$ in the endoscopic view at the distance. The average laser power density on the spot was estimated to be 170-370 $\mathrm{W} / \mathrm{cm}^{2}$, and a chicken liver was successfully coagulated by changing the laser beam irradiation angle.
\end{abstract}

\section{Introduction}

Twin-to-twin transfusion syndrome (TTTS) occurs in about $15 \%$ of monochorionic twins, caused by imbalance in the net flow of blood between them across the placental communicating vessels 12 . The mortality rate of these twins exceeds $80 \%$, and the risk of disability in the survivors is estimated to be $15-50 \%$ if untreated [3]. As a treatment for TTTS, fetoscopic laser photocoagulation (FLP) of the communicating vessels is widely accepted and the outcome showed that at least one survivor rate reaches $80 \%$.

During surgery, surgeons operate a fetoscope and a laser fiber in a sheath to navigate the laser guide-light to the communicating vessels. The laser is fired with a distance of $10 \mathrm{~mm}$ between the laser fiber tip and the vessels [5]. The operation requires skill, as it is necessary to avoid contact with the vessels or the placenta, which bleeds easily. Bleeding disrupts clear fetoscopic view, resulting in termination of the surgery. Surgeons have to stop bleeding as soon as possible. There exist other difficulties in this system. The distance is not precisely known from the fetoscopic image, and the vessels move as the mother breathes. In addition, the orientation between the fetoscopic image and the laser fiber is not easily identified. 
In related studies, a composite-type optical fiberscope with a laser fiber coaxially coupled with optical image fibers has been developed. This fiberscope makes it easier to navigate the beam to the vessels because a laser beam is transmitted to the center of the view 6]. However, surgeons have to manipulate the fiberscope. The movement of the fiberscope must be small to avoid injuring the chorion and amniotic membranes, that possibly causing preterm rupture of the membranes. A safe treatment requires a technique for steering a laser beam on arbitrary targets without moving an endoscope. Furthermore, a thin fiberscope has a limited resolution and a narrow viewing angle and requires high illumination because light transmittance of the optical fiber is small. Surgeons are concerned that high illumination will affect premature fetal eyes.

Another related study stated a laser-pointing endoscope system that can acquire intraoperative 3D geometric information using two endoscopes, one projects a laser beam and the other captures the endoscopic image [7. The projection point of the laser beam can be controlled using galvanometers. The laser source is a red diode which is easy to handle because its collimated beam is thin and the laser power is small. However, the system requires two endoscopes, which increases the invasion and the risk of complications. Furthermore, the low-powered laser doesn't suit photocoagulation. Therefore, development of an endoscope integrated with a high-powered laser irradiation function for arbitrary targets is important for treatment of TTTS.

We suggest a rigid endoscope that can transmit a laser beam to arbitrary points within the view without moving the endoscope itself. The rigid endoscope ensures a wide viewing angle and high transmittance of visible light.

\section{Materials and Methods}

\subsection{Concept of Laser Endoscope}

A rigid endoscope transmits a near-infrared laser beam for FLP, as shown in Fig. 1. Therefore, each lens of the endoscope has an anti-reflective (AR) coating for better transmission of visible and near-infrared light (VIS-NIR coating). For coaxial transmission of the laser beam, a hot mirror, which reflects near-infrared light and transmits visible light, is set between the endoscope and a camera. The beam and guide light from a laser fiber are reflected by galvano mirrors toward the hotmirror, which further directs this beam into the endoscope. The galvano mirrors control the direction of the beam at the entrance to the endoscope, then the beam is transmitted in a zigzag path around the optical axis of the endoscope and finally projected from the endoscope tip to some direction. The galvano mirrors and the camera are placed at optically symmetric points, which are equal to the eye point of the endoscope, with respect to the hot mirror to ensure a large range of the projection angle.

In clinical use, a surgeon selects a target point on the endoscopic image. the direction from the endoscope tip to the target is calculated, and the galvano mirrors rotate to appropriate angles to navigate the laser beam to the target. After confirming the guide-light spot on the image, the surgeon fires the laser. 


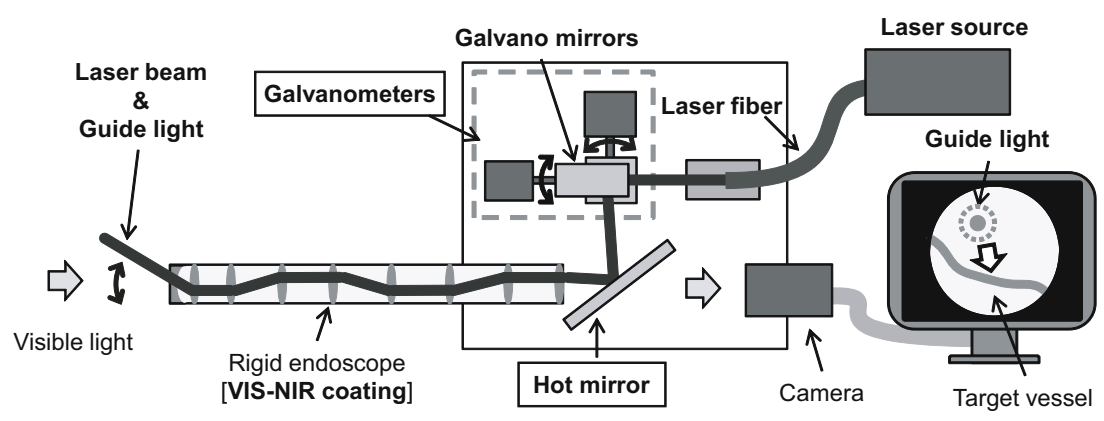

Fig. 1. Concept of the endoscope which provides an endoscopic image and transmits a laser beam coaxially to arbitrary target vessels in the endoscopic view

\subsection{Design of the Laser Endoscope}

To achieve a high laser power density in the spot, the laser spot should be small. A small spot can be achieved when the laser fiber has a small core and a small numerical aperture (NA) and the endoscope has object lenses with a small viewing angle and relay lenses with a large NA.

We used a Nd:YAG laser and a laser fiber (Fibertom 5100 / E-4070-B, Dornier MedTech) whose core diameter of $400 \mu \mathrm{m}$. The endoscope had relay lenses with an NA of about 0.12 and object lenses with a viewing angle of over 60 degrees. This design produced a spot as small as $2-3 \mathrm{~mm}$ in diameter at a distance of 10-20 $\mathrm{mm}$ from the endoscope tip with a changeable angle of the laser beam of about \pm 15 degrees. The prototype endoscope consisted of concave or convex lenses with a diameter of $6 \mathrm{~mm}$. The endoscope had an outer diameter of $7 \mathrm{~mm}$, a viewing angle of about 70 degrees and an effective length of about $150 \mathrm{~mm}$ (Fig. 2). Handling was easy because its controlling unit was small and weighed as light as $450 \mathrm{~g}$. The laser fiber tip was cooled by circulating water to protect it from damage by heat.

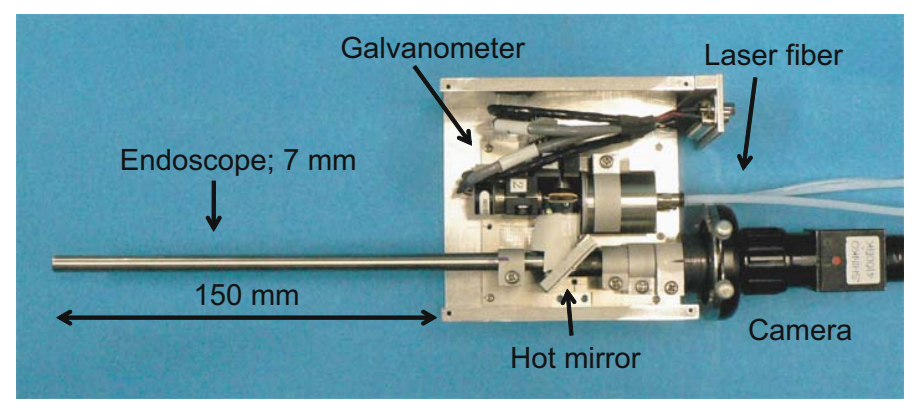

Fig. 2. Developed endoscope 


\subsection{Positioning the Laser Spot}

For controlling the laser spot to target points indicated by the surgeon on the endoscopic image, it is necessary to calibrate the coordinates of the camera (or image) and the galvano mirrors. We acquired the relationship of their coordinates to the endoscope tip.

Camera Calibration. The relationship between a point $\boldsymbol{q}$ in the coordinates of the image and a point $\boldsymbol{p}$ in the coordinates of the endoscope tip is represented by a camera model with rotation $R$, translation $\boldsymbol{t}$, scale factors $\lambda$ and $\alpha$, and principal points $u_{o}$ and $v_{o}$.

$$
\lambda\left(\begin{array}{l}
\boldsymbol{q} \\
1
\end{array}\right)=\left[\begin{array}{cccc}
\alpha_{u} & 0 & u_{o} & 0 \\
0 & \alpha_{v} & v_{o} & 0 \\
0 & 0 & 1 & 0
\end{array}\right]\left[\begin{array}{cc}
R & \boldsymbol{t} \\
O_{1 \times 3} & 1
\end{array}\right]\left(\begin{array}{c}
\boldsymbol{p} \\
1
\end{array}\right)
$$

We took fifty different postures of a checker pattern whose pitch was known, and calculated the coordinates and the distortion parameters by a flexible view technique [8. The technique also provided the coordinates of the checker pattern to the camera. In addition, we used an optical tracking system (Micron Tracker 2, Claron Technology Inc.) to acquire the relationship between the camera and the endoscope tip. The optical tracker obtained the marker coordinates at the endoscope tip and the sheet where the checker pattern was written. The relationship between the checker pattern and the marker was known. Therefore, the relationship between the camera and the endoscope tip was calculated.

Galvano Mirror Calibration. The two galvano mirrors were located at different distances from the endoscope. We used a model, similar to the camera model, to calibrate each coordinate of the $\mathrm{X}$ - and $\mathrm{Y}$-axes independently. The relationship between optical angle $\theta$ of the mirror and laser spot $\boldsymbol{p}$ in the coordinates of the endoscope tip is represented as below.

$$
\lambda\left(\begin{array}{c}
\tan \theta \\
1
\end{array}\right)=C_{\text {in }}\left[\begin{array}{cc}
R & \boldsymbol{t} \\
O_{1 \times 3} & 1
\end{array}\right]\left(\begin{array}{c}
\boldsymbol{p} \\
1
\end{array}\right)
$$

where

$$
C_{i n-X}=\left[\begin{array}{cccc}
\alpha_{X} & 0 & u_{o} & 0 \\
0 & 0 & 1 & 0
\end{array}\right], C_{i n-Y}=\left[\begin{array}{cccc}
0 & \alpha_{Y} & v_{o} & 0 \\
0 & 0 & 1 & 0
\end{array}\right]
$$

A data-set of relations between the optical angles of the galvano mirrors and the position of laser spots was required to calculate the parameters. We ablated sheets fixed at some distances from the endoscope tip by rotating the galvano mirrors. We used the tracking system and acquired the coordinates of the markers on the sheets and the endoscope tip. The sheets were scanned and the relations of the ablated spots and the marker were recorded. Then, the positions of the spots were transformed into the coordinate of the endoscope tip. The transformation matrix from the target point to the angle of the mirrors was calculated by a technique similar to the direct linear transformation method after normalization of the data set [910]. 


\section{Experiments and Results}

We evaluated the transmission efficiency of laser power, the spot size, positioning accuracy of the spot and irradiated a chicken liver to test feasibility of the system.

\subsection{Transmission Efficiency of Laser Power}

We measured the power of the laser beam on the optical axis through the endoscope using a power meter (30(150)A-HE/NOVA, OPHIR) to evaluate the transmission efficiency. The laser power was set as 10-50 W at source. During measurement, the cooling water flowed at a speed of $6 \mathrm{ml} / \mathrm{s}$. We didn't measure the power from the laser fiber directly at a source laser power of over 30 $\mathrm{W}$ because of the danger of breaking the fiber tip. Therefore, the transmission efficiency was calculated with the source power over $30 \mathrm{~W}$.

The maximum efficiency was $39.1 \%$ at a source power of $10 \mathrm{~W}$ (Table 1). The efficiency decreased as the power increased. The maximum laser power from the endoscope was $16.1 \mathrm{~W}$ at a source power of $50 \mathrm{~W}$.

Table 1. Transmission efficiency of the laser endoscope

\begin{tabular}{cccc}
\hline Source & Laser fiber & Endoscope & Efficiency \\
\hline $10 \mathrm{~W}$ & $10.9 \pm 0.1 \mathrm{~W}$ & $4.3 \pm 0.2 \mathrm{~W}$ & $39.1 \%$ \\
$20 \mathrm{~W}$ & $20.5 \pm 0.1 \mathrm{~W}$ & $7.9 \pm 0.1 \mathrm{~W}$ & $38.6 \%$ \\
$30 \mathrm{~W}$ & - & $11.1 \pm 0.1 \mathrm{~W}$ & $36.9 \%$ \\
$40 \mathrm{~W}$ & - & $13.6 \pm 0.1 \mathrm{~W}$ & $34.1 \%$ \\
$50 \mathrm{~W}$ & - & $16.1 \pm 0.1 \mathrm{~W}$ & $32.2 \%$ \\
\hline
\end{tabular}
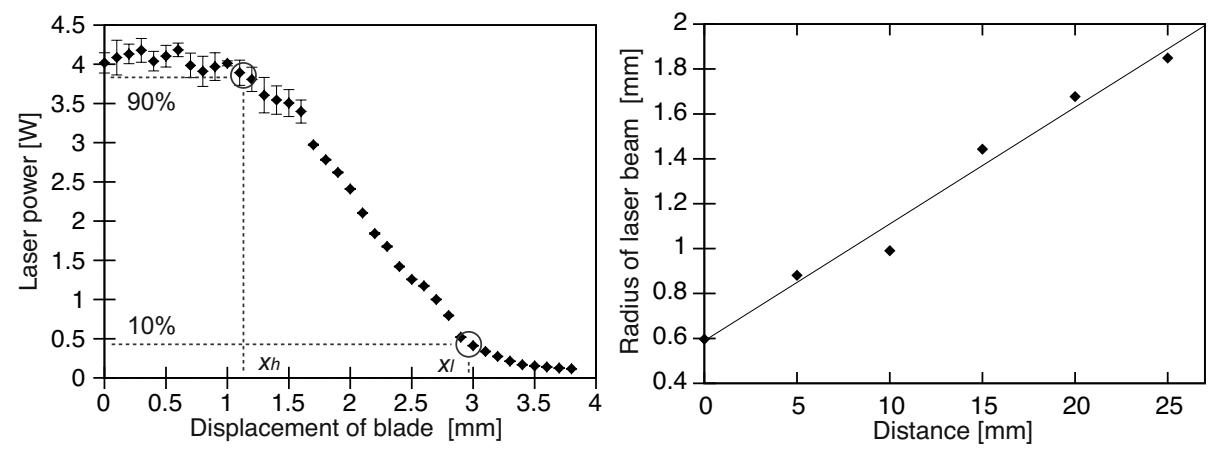

Fig. 3. Knife-edge method for measuring laser beam radius. Left: Laser power with blade displacement at a distance of $15 \mathrm{~mm}$. Right: Laser beam radius from the endoscope tip. 

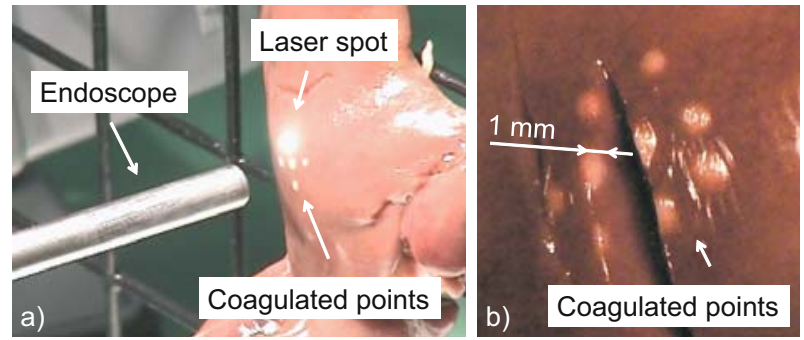

Fig. 4. Laser irradiation of a chicken liver. a) Changing irradiation angle; b) coagulated points on the liver.

\subsection{Laser Spot Size}

We evaluated the laser beam radius on the optical axis through the endoscope by the knife-edge method. A knife was set to cut the laser beam at a right angle and moved in $0.1 \mathrm{~mm}$ steps. The power meter behind the knife measured the portion of the laser not obstructed by the knife, and we captured the power shift from the displacement of the knife. The laser beam radius $d / 2$ was calculated from displacement $x_{h}, x_{l}$ at 90 and $10 \%$ of the maximum power (Fig. 3.left)[1].

$$
d / 2=1.561 \times\left|x_{h}-x_{l}\right| / 2
$$

At a distance of $10-20 \mathrm{~mm}$ from the endoscope, the radius was about $1.1-1.6$ $\mathrm{mm}$, which equals $2.2-3.2 \mathrm{~mm}$ in diameter, as shown in the right side of Fig. 3 .

\subsection{Positioning Accuracy}

We evaluated accuracy in positioning the laser spot. Sheets which have a grid with a pitch of $5 \mathrm{~mm}$ were placed at a distance of $10-20 \mathrm{~mm}$ from the endoscope tip. We pointed to the grid points on the endoscopic image and fired the laser. After irradiation, we scanned the sheet and measured the distance between the target and the actually irradiated points. The errors were $0.4 \pm 0.3 \mathrm{~mm}$ at a 10 $\mathrm{mm}$ distance, $0.5 \pm 0.4 \mathrm{~mm}$ at a $15 \mathrm{~mm}$ distance, and $0.5 \pm 0.6 \mathrm{~mm}$ at a $20 \mathrm{~mm}$ distance from the endoscope tip. The errors were mostly smaller than $1.0 \mathrm{~mm}$ in the view except on the upper side.

\subsection{In Vitro Irradiation to Phantom}

We irradiated a chicken liver with the laser to evaluate the laser endoscope for photocoagulation. A chicken liver is similar to a placenta in terms of the amount of blood. The liver was fixed at a distance of 10-15 $\mathrm{mm}$ from the endoscope and the laser source power was set as $50 \mathrm{~W}$. We irradiated some points on the liver for $3 \mathrm{sec}$ by changing the irradiation angle (Fig. 4.a). All the irradiated points were coagulated and the depths of coagulation were about $1 \mathrm{~mm}$ (Fig. 4 $\mathrm{b}$ ). 


\section{Discussion}

The maximum efficiency was nearly equal to the ideal value of $41 \%$ based on the transmission and reflection rates of the optical elements. Reduction in the transmission efficiency from the ideal value was due to dirt on the elements and power loss from the small galvano mirrors that could not use the full power of laser beam. At higher power, bubbles were generated around the laser fiber tip due to the cooling water, which diffused the beam. This resulted in loss of efficiency.

The laser spot diameter at a distance of $10-20 \mathrm{~mm}$ was $2.2-3.2 \mathrm{~mm}$, which was suitable for coagulating placental vessels $1-2 \mathrm{~mm}$ in diameter. The distance is further than that of conventional treatment, which reduces the risk of contact with the placenta.

The laser endoscope achieved sufficiently high accuracy in positioning the laser spot because most of the errors were smaller than the diameter of the placental vessels in the target period for the surgery. In ordinary surgery, accuracy depends on the surgeon's skill, and there is risk in manipulating a fiberscope and a laser fiber. This risk is eliminated in the laser endoscope because the laser spot position can be controlled without moving the endoscope itself. The surgeon simply points to targets on the endoscopic image. This capability makes the surgery easier and safer.

Photocoagulation within a few seconds requires the power density of 100-1000 $\mathrm{W} / \mathrm{cm}^{2}\left[1213\right.$. The average power density of the spot was $170-370 \mathrm{~W} / \mathrm{cm}^{2}$ from $16.1 \mathrm{~W}$ at the source power of $50 \mathrm{~W}$, that meets the minimum requirement. Furthermore, the endoscope successfully performed photocoagulation of a chicken liver.

For clinical use, we plan to miniaturize the endoscope by using smaller lens. A small laser spot can be achieved by an appropriate design. For higher transmittance, we optimize the selection of a laser and an AR coating and reduce the amount of lenses because the main cause of the transmission reduction is reflection of the laser beam on the surface of lenses.

\section{Conclusion}

We developed a prototype of a rigid endoscope that steers a laser beam on any point in the endoscopic view without moving the endoscope itself, mainly for treatment of TTTS. The maximum laser transmission efficiency was $39 \%$. The spot diameter was $2.2-3.2 \mathrm{~mm}$ at a distance of $10-20 \mathrm{~mm}$, which reduces the risk of contact with placenta. The positioning accuracy was mostly within 1.0 $\mathrm{mm}$ in the endoscopic view at the distance. The average laser power density at a spot was estimated to be $170-370 \mathrm{~W} / \mathrm{cm}^{2}$, and we successfully coagulated a chicken liver by changing the irradiation angle.

Acknowledgments. This study was partly supported by Japan Society for the Promotion of Science (JSPS, 21.9099) and Ministry of Education, Culture, Sports, Science and Technology (MEXT). 


\section{References}

1. Sebire, N.J., Souka, A., Skentou, H., Geerts, L., Nicolaides, K.H.: Early prediction of severe twin-to-twin transfusion syndrome. Human Reproduction 15(9), 2008$2010(2000)$

2. Deprest, J., Jani, J., Lewi, L., Ochsenbein-Kölble, N., Cannie, M., Doné, E., Roubliova, X., Mieghem, T.V., Debeer, A., Debuck, F., Sbragia, L., Toelen, J., Devlieger, R., Lewi, P., Velde, M.V.: Fetoscopic surgery: Encouraged by clinical experience and boosted by instrument innovation. Seminars in Fetal and Neonatal Medicine 11, 398-412 (2006)

3. Yamamoto, M., Murr, L.E., Robyr, R., Leleu, F., Takahashi, Y., Ville, Y.: Incidence and impact of perioperative complications in 175 fetoscopy-guided laser coagulations of chorionic plate anastomoses in fetofetal transfusion syndrome before 26 weeks of gestation. Am. J. Obste. Gynecol. 193, 1110-1116 (2005)

4. Rossi, A.C., D'Addario, V.: Laser therapy and serial amnioreduction as treatment for twin-twin transfusion syndrome: a metaanalysis and review of literature. Am. J. Obste. Gynecol. 198, 147-152 (2008)

5. Ville, Y., Hecher, K., Ogg, D., Warren, R., Nicolaides, K.: Successful outcome after $\mathrm{Nd}$ :YAG laser separation of chorioangiopagus-twin under sonoendoscopic control. Ultrasound Obstet. Gynecol. 2, 429-431 (1992)

6. Oka, K., Naganawa, A., Yamashita, H., Nakamura, T., Chiba, T.: Composite-Type Optical Fiberscope for Laser Surgery for Twin-to-Twin Transfusion Syndrome. In: Dohi, T., Sakuma, I., Liao, H. (eds.) MIAR 2008. LNCS, vol. 5128, pp. 251-259. Springer, Heidelberg (2008)

7. Hayashibe, M., Nakamura, Y.: Laser-Pointing Endoscope System for IntraOperative 3D Geometric Registration. In: Proceedings of the 2001 IEEE International Conference on Robotics and Automation, vol. 2, pp. 1543-1548 (2001)

8. Zhang, Z.: A Flexible New Technique for Camera Calibration. IEEE Transaction on Pattern Analysis and Machine Intelligence 22(11), 1330-1334 (2000)

9. Abdel-Aziz, Y.I., Karara, H.M.: Direct linear transformation from comparator coordinates into object space coordinates. In: Proceedings of the ASP/UI Symposium on Close-Range Photogrammetry, pp. 1-18 (1971)

10. Marzan, G.T., Karara, H.M.: A computer program for direct linear transformation solution of the collinearity condition, and some applications of it. In: Proceedings of the Symposium on Close-Range Photogrammetric Systems, pp. 420-476 (1975)

11. Siegman, A.E., Sasnett, M.W., Johnston Jr., T.F.: Choice of Clip Levels for Beam Width Measurements Using Knife-Edge Techniques. IEEE Journal of Quantum Electronics 27(4), 1098-1104 (1991)

12. Boulnois, J.-L.: Photophysical Processes in Recent Medical Laser Developments: a Review. Lasers in Medical Science 1, 47-66 (1986)

13. Dörschel, K., Brodzinski, T.: Proposal for dosimetry of non-ionizing radiation. Lasers in Medical Science 4(1), 329-340 (1989) 